\title{
Ophthalmic viscosurgical device backflow into cartridge during intraocular lens insertion using injectors
}

This article was published in the following Dove Press journal:

Clinical Ophthalmology

31 January 2014

Number of times this article has been viewed

\section{Kazuki Matsuura' Yoshitsugu Inoue ${ }^{2}$ \\ 'Nojima Hospital, ${ }^{2}$ Tottori University, Kurayoshi City, Tottori, Japan}

Correspondence: Kazuki Matsuura Nojima Hospital, 27I4-I Sesaki-machi, Kurayoshi City, Tottori 682-0863, Japan Tel +8I85822 623 I

$\mathrm{Fax}+81858226843$

Email matsu224@ncn-k.net
Background: The purpose of this study was to assess the risk of intraocular contamination caused by intraocular lens (IOL) insertion with injectors by observing the dynamics of an ophthalmic viscosurgical device (OVD).

Methods: Each type of injector was equipped with a colored OVD and IOL, and a $2 \mathrm{~mm}$ length from the tip of the cartridge was replaced with a colored OVD. The various combinations of IOLs and injectors used were: a three-piece shaped IOL, VA60BBR + TypeE1 (HOYA incision size $2.5 \mathrm{~mm}$; group $\mathrm{A}, \mathrm{n}=5$ ); a single-piece IOL, 251+ iSert micro, preloaded (HOYA, incision size $2.2 \mathrm{~mm}$; group $\mathrm{G}, \mathrm{n}=5$ ); and a single-piece IOL, SN6CWS preloaded (Alcon, incision size $2.7 \mathrm{~mm}$; group $\mathrm{C}, \mathrm{n}=5$ ).

Results: In group A, the intraocular OVD instantly flowed backward into the injector, whereas the colored OVD was pushed backward deep inside the cartridge without flowing into the eye. In group B, the backflow of the intraocular OVD into the injector was limited, resulting in the influx of a large amount of the colored OVD into the eye along with the IOL. In group C, as in group A, a large amount of the intraocular OVD flowed backward into the injector. Consequently, a small amount of the colored OVD flowed into the eye.

Conclusion: The tip of the injector and OVD could be contaminated because the surgical field cannot be completely sterile, even after preoperative disinfection. Our experiments revealed that OVD backflow into the injector cavity occurs during IOL insertion, and this phenomenon may have minimized intraocular contamination. However, small-diameter cartridges along with plate-type haptics allow insufficient OVD backflow, resulting in intraocular influx of the contaminated OVD. Surgeons have to be notified that intraoperative bacterial contamination can occur even after IOL insertion using injectors.

Keywords: intraocular lens insertion, endophthalmitis, injector, backflow, ophthalmic viscosurgical device

\section{Introduction}

Endophthalmitis can be induced by pathogens that are transferred into the eye during surgery. Although intraocular contamination frequently results after intraocular lens (IOL) insertion, the mechanism and timing of contamination are not well understood. With regard to intraocular contamination, small incisions and the use of injectors are believed to be safe. IOL insertion using injectors has been shown to decrease the incidence of endophthalmitis because they allow limited contact between the IOL and the conjunctival flora. ${ }^{1}$ However, previous studies that compared anterior chamber bacterial contamination with IOL implantation using injectors or forceps failed to detect significant differences. ${ }^{2,3}$ 
On February 20, 2013, there was a rare incident in Japan, wherein a self-imposed control on sales of the iSert micro 251 preloaded acrylic IOL system (HOYA Tokyo, Japan) was enforced because of a suspected high risk of endophthalmitis. On April 2, 2013, a medical device alert for HOYA single-piece IOLs was issued in the UK because higher than expected rates of inflammation and endophthalmitis were observed after implantation. Following investigation by the manufacturer, it was determined that the lenses contained traces of residual foreign particles. The manufacturer, however, has been unable to definitively determine if these particles were linked to the adverse events. Currently, a clear mechanistic link between endophthalmitis, IOLs, and injectors is lacking. Endophthalmitis caused by infection is generally thought to be caused by bacteria introduced by an ophthalmic viscosurgical device (OVD) during IOL insertion. Secretion and debris from the meibomian gland are often observed in the surgical field, which means that the tip of the injector and OVD may be contaminated by bacteria in the surgical field. This study aimed to examine whether OVD in the tip of the injector could flow into the eye and examine the potential differences in OVD dynamics in the eye.

\section{Materials and methods}

Water-based red ink was mixed with the OVD (Opegan $\mathrm{Hi}^{\circledR}$, Santen; Osaka, Japan) to create a colored OVD. The Opegan $\mathrm{Hi}$ is purified sodium hyaluronate with a molecular weight of $250 \times 10^{3} \mathrm{Da}$, and is a commonly used OVD in Japan. Each type of injector was equipped with an OVD and IOL, and a $2 \mathrm{~mm}$ length from the tip of cartridge was subsequently replaced with the colored OVD. This enabled us to recreate and visually verify the conditions under which the injector tips were contaminated in the surgical field during IOL insertion with injectors. Lens removal was performed using normal ultrasonic surgery by placing a $2.7 \mathrm{~mm}, 2.5 \mathrm{~mm}$, or $2.2 \mathrm{~mm}$ incision into an excised porcine eye. The Infiniti ${ }^{\circledR}$ vision system (Alcon, Fort Worth, TX, USA) was used to remove the lens. The anterior chamber was filled with the OVD, and the IOL was inserted using an injector after adjusting the intraocular pressure to approximately $15-20 \mathrm{mmHg}$ using a Schiotz tonometer. The various combinations of IOLs (Figure 1) and injectors used in this study were: a threepiece shaped IOL, VA60BBR + TypeE1 (HOYA incision size $2.5 \mathrm{~mm}$; group $\mathrm{A}, \mathrm{n}=5$ ); a single-piece IOL, 251+ iSert micro, preloaded (HOYA incision size $2.2 \mathrm{~mm}$; group G, $\mathrm{n}=5$ ); and a single-piece IOL, SN6CWS preloaded (Alcon, incision size $2.7 \mathrm{~mm}$; group $\mathrm{C}, \mathrm{n}=5$ ).

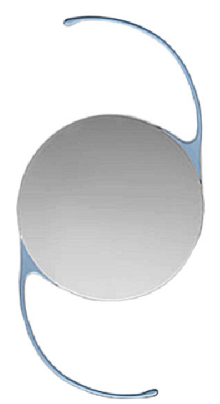

A: HOYA BBR

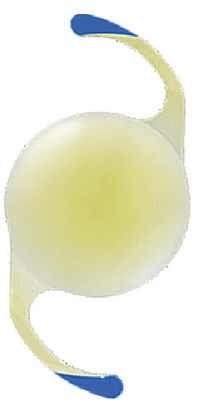

B: HOYA 251

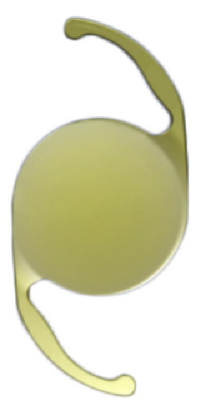

C: Alcon SN6CWS
Figure I IOLs used in the study. (A) Three-piece-shaped IOL with filament-shaped haptics. This IOL, which has an acrylic optic and polymethylmethacrylate haptic, is a common three-piece-shaped IOL. According to HOYA however, it is not classified as a typical three-piece IOL, but as a two-material single-piece IOL because the optic and haptic are polymerized. (B) Single-piece-shaped IOL with plate-type haptics. (C) Single-piece-shaped IOL with plate-type haptics.

Abbreviation: IOL, intraocular lens.

\section{Results}

In group A (three-piece shaped IOL with filament-type haptics, incision size $2.5 \mathrm{~mm}$ ), the intraocular OVD instantly flowed backward into the injector when the injector tip was inserted into the incision wound, causing the colored OVD to be pushed backward deep inside the cartridge without being introduced into the eye (Figure 2A-2E).

In group B (single-piece IOL with plate-type haptics, incision size $2.2 \mathrm{~mm}$ ), the intraocular OVD similarly flowed backward into the injector when the injector tip was inserted into the incision; however, the amount of flow was limited. As a result, the colored OVD was not pushed backward deep inside the cartridge, and a large amount of the colored OVD enveloping the entire IOL was introduced into the eye along with the IOL (Figure 3A-3E). The same OVD dynamics were observed in all five tests in groups A and B.

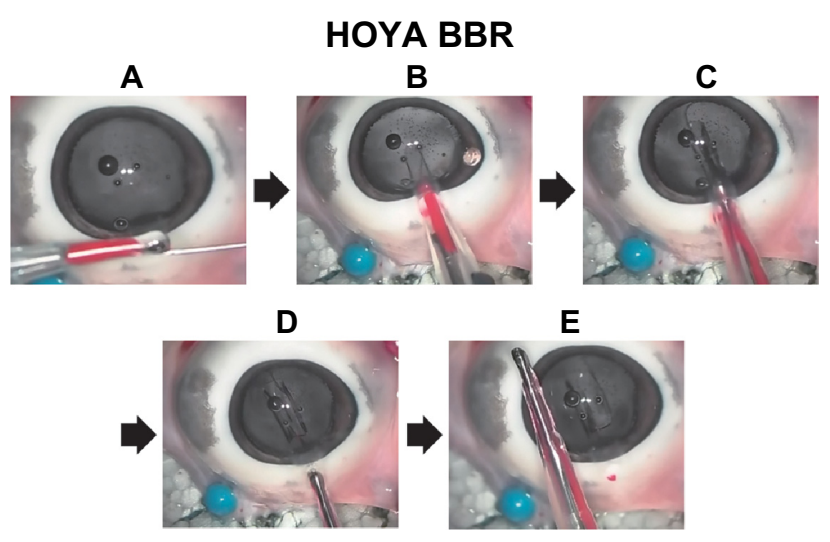

Figure 2 Three-piece IOL+2.5 mm injector HOYA VA60 BBR.

Notes: The tip of cartridge was replaced with colored OVD (A). Through the gap created by the folded IOL (B), the colored OVD is pushed backward deep inside the cartridge without being introduced into the eye $(\mathbf{C}, \mathbf{D}, \mathbf{E})$.

Abbreviations: IOL, intraocular lens; OVD, ophthalmic viscosurgical device. 


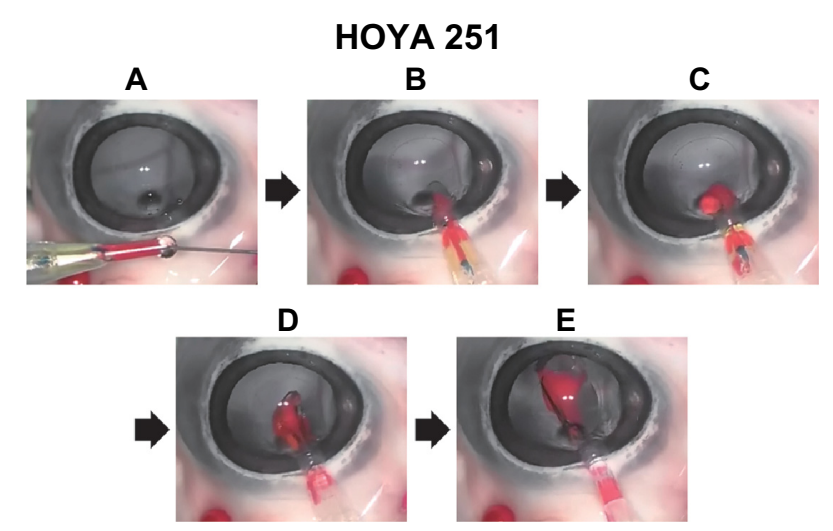

Figure 3 Single-piece $I O L+$ injector for minimum $(2.2 \mathrm{~mm}$ ) incision, $25 \mathrm{I}+\mathrm{iSert}$ micro. Notes: The tip of cartridge was replaced with colored OVD (A).The posterior platetype haptic, which has a relatively wide tip, closed the gap created by the folded IOL (B). Therefore, the OVD-IOLexchange became inadequate, resulting in a large amount of the colored OVD being introduced into the eye with the IOL (C, D, E).

Abbreviations: IOL, intraocular lens; OVD, ophthalmic viscosurgical device.

In group C (single-piece IOL with plate-type haptics, incision size $2.7 \mathrm{~mm}$ ), a large amount of the intraocular OVD flowed backward into the injector, similar to that observed in group A. Furthermore, there was a case in which the colored OVD enveloping a plate-type haptic IOL was introduced into the eye, although the amount was much smaller than that in group B (Figure 4A-4F).

\section{Discussion}

It has been reported that bacteria are present in $10 \%-40 \%$ of eyes immediately after surgery, ${ }^{4,5}$ and a majority of postoperative endophthalmitis cases are thought to result from intraoperative infections. In fact, bacterial genes isolated from endophthalmitis were consistent with indigenous bacterial genes in the conjunctival sac. ${ }^{6}$ In endophthalmitis, bacteria from the operative field are thought to inadvertently

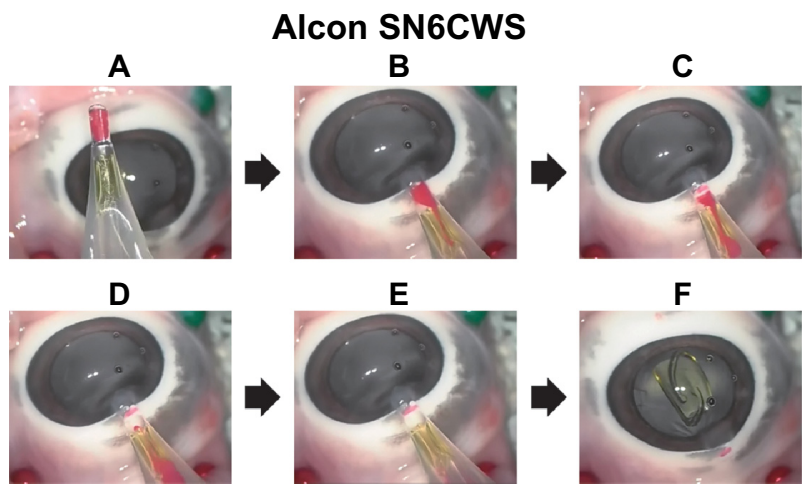

Figure 4 The single-piece $1 \mathrm{OL}+2.7 \mathrm{~mm}$ injector, SN6CWS.

Notes: The tip of cartridge was replaced with colored OVD(A). Although the anterior and posterior plate-type haptics were tucked in, a reasonable gap remained inside the cartridge because it was wide $(2.7 \mathrm{~mm})$ and the haptic was relatively narrow (B). Therefore, a large amount of intraocular OVD flowed back into the injector, similar to that observed in group A (C, D, E, F). OVD was not introduced into the eye (F). Abbreviations: IOL, intraocular lens; OVD, ophthalmic viscosurgical device. enter and remain in the eye as a result of the insertion and removal of surgical instruments. Therefore, IOL insertion is considered to be a particularly important cause of intraocular contamination. Because the surgical field cannot be completely sterile, OVD in the tip of the injector would be contaminated and flow into the eye with the IOL. Even if bacteria are only temporarily present in the anterior chamber, a majority are discharged by the flow of aqueous humor. Moreover, the presence of immune cells such as macrophages and the antibacterial action of eye drops would likely eliminate any bacteria. However, the ability of antibacterial compounds to act on the region posterior to the IOL is very poor. Suzuki et al demonstrated that the bacteria left behind in this region can break through the posterior capsule and invade the vitreous body, thereby causing endophthalmitis. ${ }^{7}$ Therefore, IOL contamination leads to contamination of the region inside the capsular bag, resulting in endophthalmitis.

From this perspective, the use of microincision injectors should decrease the incidence of endophthalmitis. Cornut et al inserted an IOL using an injector and obtained aqueous fluid samples at the end of surgery before OVD removal. Although the bacterial detection rate $(1.8 \%)$ was lower than that reported previously, it was indicated that bacteria would flow into the eye even if injectors were used. ${ }^{8}$

We presumed that the OVD in the cartridge was injected into the eye while adherent to the IOL, but this was not the case. A significant finding was that in group A, the intraocular OVD flowed backward into the cartridge as soon as the injector tip was inserted into the incision; this introduced the IOL alone into the eye without the surrounding OVD. In other words, an exchange of the OVD and IOL occurred between the injector and the eye. Gotoh et al reported that intraocular pressure temporarily increases to approximately 40-100 mmHg during IOL insertion with injectors. ${ }^{9}$

When the pressure increases, the intraocular OVD instantly flows backward into the injectors because it has no other escape route. As observed in group A, when a three-piece shaped IOL with a filament-type haptics was used in the recommended $2.5 \mathrm{~mm}$ injector, there remained a considerable gap in the cross-sectional area of the injector occupied by the IOL and the loop; therefore, a considerable amount of IOL-OVD exchange was performed to ensure a favorable pathway for the OVD. In this case, the large amount of sterile OVD previously injected into the eye instantly flowed backward into the cartridge and rinsed the IOL with a wave of sterile OVD, resulting in insertion of the IOL alone. Alternatively, as observed in group B, when a single-piece IOL with a plate-type haptic was used in the recommended 
$2.2 \mathrm{~mm}$ injector, the plate-type haptics as well as the IOL were tucked into the originally narrow cavity and the OVD pathway was mostly blocked. In addition, the relatively wide 251 haptic further worsened this situation. The decreased backflow into the OVD cartridge resulted in insufficient IOL-OVD exchange; consequently, the IOL was introduced into the eye along with the colored OVD. In contrast, in group C, a good amount of the OVD flowed backward and a small amount of the colored OVD was introduced into the anterior chamber in only one of the five eyes, despite the use of tucked-in plate-type haptics. This may be caused by the presence of a wide OVD pathway because the inner diameter of the cartridge is relatively wide and the SN6CWS haptic is relatively narrow.

It was suggested that clean injectors would be indirectly contaminated by the backflow of the OVD. The frequency and degree may be slight because the OVD and IOL inside the injector will not directly touch the surgical field. However, as Cornut et al mentioned, perhaps the $1.8 \%$ positive reaction rate of bacteria was underestimated. ${ }^{8}$

Our observation indicated that the IOL was inserted into the eye and enveloped by the colored OVD. Therefore, planktonic bacteria may not be present in the aqueous humor obtained just after surgery. Contaminating bacteria may firmly adhere to the IOL and remain in the capsular bag, resulting in endophthalmitis. ${ }^{10}$

Our observation can explain, at least partially, the high incidence of endophthalmitis using the HOYA iSert micro 251. Currently, the use of plate-type haptic IOLs is increasing worldwide, and $97 \%$ of surgeons in the US reportedly choose acrylic single-piece IOLs. ${ }^{11}$ Furthermore, excellent progress is being made with injectors, and injectors that can handle smaller incisions are undoubtedly being developed. Nevertheless, the risk of endophthalmitis, which developed with the HOYA iSert micro 251 system in Japan, must also be considered for products made by other manufacturers and their use by surgeons implanting such IOLs.

Our experiments revealed that OVD backflow into the injector cavity occurs during IOL insertion, and this may have minimized intraocular contamination. However, smalldiameter cartridges used together with plate-type haptics do not allow sufficient OVD backflow, resulting in the intraocular influx of contaminated OVD. Surgeons should be notified that intraoperative bacterial contamination can occur even after IOL insertion using preloaded injectors.

With regard to the prevention of endophthalmitis, the injectors should not be overly trusted. Therefore, the use of additional prophylactic measures against endophthalmitis, such as intracameral antibiotics and/or intraoperative disinfection using povidone iodine, seems to be justified.

\section{Disclosure}

The IOLs used in this study were supplied by Alcon and HOYA. No funding of any kind from any company was received to perform the current study.

\section{References}

1. Mayer E, Cadman D, Ewings P, et al. A 10-year retrospective survey of cataract surgery and endophthalmitis in a single eye unit: injectable lenses lower the incidence of endophthalmitis. Br J Ophthalmol. 2003;87(7):867-869.

2. Bausz M, Fodor E, Resch MD, Kristóf K. Bacterial contamination in the anterior chamber after povidone-iodine application and the effect of the lens implantation device. J Cataract Refract Surg. 2006;32(10): 1691-1695.

3. Weindler J, Spang S, Jung WK, Ruprecht KW. Bacterial anterior chamber contamination with foldable silicone lens implantation using a forceps and an injector. J Cataract Refract Surg. 1996;22 Suppl 2: $1263-1266$.

4. John T, Sims M, Hoffman C. Intraocular bacterial contamination during sutureless, small incision, single-port phacoemulsification. J Cataract Surg. 2000;26(12):1786-1791.

5. Tervo T, Ljungberg P, Kautiainen T, et al. Prospective evaluation of external ocular microbial growth and aqueous humor contamination during cataract surgery. J Cataract Refract Surg. 1999;25(1):65-71.

6. Speaker MG, Milch FA, Shah MK, Eisner W, Kreiswirth BN. Role of external bacterial flora in the pathogenesis of acute postoperative endophthalmitis. Ophthalmology. 1991;98(5):639-649.

7. Suzuki T, Wada T, Kozai S, Ike Y, Gilmore MS, Ohashi Y. Contribution of secreted proteases to the pathogenesis of postoperative Enterococcus faecalis endophthalmitis. J Cataract Refract Surg. 2008;34(10): 1776-1784.

8. Cornut PL, Vandenesch F, Lina G, et al. Bacterial contamination rate of the anterior chamber during cataract surgery using conventional culture and eubacterial PCR. Eur J Ophthalmol. 2010;20(2):365-369.

9. Gotoh N, Mastushima H, Senoo T. Changes in anterior chamber pressure during injection of different types intraocular lenses from micro incision. Poster presented at the ASCRS Symposium on Cataract, IOL and Refractive Surgery, Chicago, IL, USA, April 20-24, 2012. Available from: http://ascrs2012.abstractsnet.com/abstracthome.wcs. Accessed November 22, 2013.

10. Baillif S, Casoli E, Marion K, et al. A novel in vitro model to study staphylococcal biofilm formation on intraocular lenses under hydrodynamic conditions. Invest Ophthalmol Vis Sci. 2006;47(8):3410-3416.

11. Greenberg PB, Havnaer A, Oetting TA, Garcia-Ferrer FJ. Cataract surgery practice patterns in the United States Veterans Health Administration. J Cataract Refract Surg. 2012;38(4):705-709. 
Clinical Ophthalmology

\section{Publish your work in this journal}

Clinical Ophthalmology is an international, peer-reviewed journal covering all subspecialties within ophthalmology. Key topics include: Optometry; Visual science; Pharmacology and drug therapy in eye diseases; Basic Sciences; Primary and Secondary eye care; Patien Safety and Quality of Care Improvements. This journal is indexed on

PubMed Central and CAS, and is the official journal of The Society of Clinical Ophthalmology (SCO). The manuscript management system is completely online and includes a very quick and fair peer-review system, which is all easy to use. Visit http://www.dovepress.com/ testimonials.php to read real quotes from published authors. 